\title{
A Final Solution to the Mind-Body Problem by Quantum Language
}

\author{
Shiro Ishikawa \\ Department of Mathematics, Faculty of Science and Technology, Keio University, Yokohama, Japan \\ Email: ishikawa@math.keio.ac.jp
}

How to cite this paper: Ishikawa, S. (2017) A Final Solution to the Mind-Body Problem by Quantum Language. Journal of Quantum Information Science, 7, 48-56. https://doi.org/10.4236/jqis.2017.72005

Received: April 17, 2017

Accepted: May 21, 2017

Published: May 24, 2017

Copyright $\odot 2017$ by author and Scientific Research Publishing Inc. This work is licensed under the Creative Commons Attribution International License (CC BY 4.0).

http://creativecommons.org/licenses/by/4.0/

\section{c) (i) Open Access}

\begin{abstract}
Recently we proposed "quantum language", which was not only characterized as the metaphysical and linguistic turn of quantum mechanics but also the linguistic turn of Descartes = Kant epistemology. And further we believe that quantum language is the only scientifically successful theory in dualistic idealism. If this turn is regarded as progress in the history of western philosophy (i.e., if "philosophical progress" is defined by "approaching to quantum language"), we should study the linguistic mind-body problem more than the epistemological mind-body problem. In this paper, we show that to solve the mind-body problem and to propose "measurement axiom" in quantum language are equivalent. Since our approach is always within dualistic idealism, we believe that our linguistic answer is the only true solution to the mindbody problem.
\end{abstract}

\section{Keywords}

History of Western Philosophy, Quantum Language, Progress, Mind-Body Problem, Linguistic Copenhagen Interpretation

\section{Preparations: Outline of Quantum Language}

If readers are not familiar with quantum language, it may be recommended to skip Sections 1.1 and 1.2 (Axioms 1 and 2) and start reading from Section 1.3.

Following refs. [1]-[8] (where [1] [2] may be simple and easy, also, all of quantum language at the present time is summarized in refs. [7] [8]), we shall review quantum language (i.e., the linguistic Copenhagen interpretation of quantum mechanics, or measurement theory), which has the following form:

$$
\underset{\text { (=measurement theory })}{\frac{\text { Quantumlangage }}{\text { (Axiom 1) }}}=\underset{\text { (Axiom 2) }}{\text { measurement }}+\frac{\text { causality }}{\text { linguistic(Copenhagen )interpretation }}
$$

where Axioms 1 and 2 are essential. I believe that quantum language is the only 
scientifically successful theory in dualistic idealism, where we assume, throughout this paper, that "idealism" = "metaphysics" = "a discipline that cannot be verified by experiment". For example, mathematics is a kind of metaphysics; however, it is not in dualism. Also, quantum mechanics is usually considered to be in dualistic realism.

\subsection{Mathematical Preparations}

Consider an operator algebra $B(H)$ (i.e., an operator algebra composed of all bounded linear operators on a Hilbert space $H$ with the norm $\left.\|F\|_{B(H)}=\sup _{\|u\|_{H}=1}\|F u\|_{H}\right)$. Let $\mathcal{A}(\subseteq B(H))$ be a $C^{*}$-algebra, which is, for simplicity, assumed to have the identity $I$. Let $\mathcal{A}^{*}$ be the dual Banach space of $\mathcal{A}$. That is, $\mathcal{A}^{*}=\{\rho \mid \rho$ is a continuous linear functional on $\mathcal{A}\}$, and the norm $\|\rho\|_{\mathcal{A}^{*}}$ is defined by $\sup \left\{|\rho(F)| \mid F \in \mathcal{A}\right.$ such that $\left.\|F\|_{\mathcal{A}}\left(=\|F\|_{B(H)}\right) \leq 1\right\}$. Define the mixed state $\rho\left(\in \mathcal{A}^{*}\right)$ such that $\|\rho\|_{\mathcal{A}^{*}}=1$ and $\rho(F) \geq 0$ for all $F \in \mathcal{A}$ such that $F \geq 0$. And define the mixed state space $\mathfrak{S}^{m}\left(\mathcal{A}^{*}\right)$ such that

$$
\mathfrak{S}^{m}\left(\mathcal{A}^{*}\right)=\left\{\rho \in \mathcal{A}^{*} \mid \rho \text { is a mixed state }\right\} .
$$

A mixed state $\rho\left(\in \mathfrak{S}^{m}\left(\mathcal{A}^{*}\right)\right)$ is called a pure state if it satisfies that " $\rho=\theta \rho_{1}+(1-\theta) \rho_{2}$ for some $\rho_{1}, \rho_{2} \in \mathfrak{S}^{m}\left(\mathcal{A}^{*}\right)$ and $0<\theta<1$ " implies “ $\rho=\rho_{1}=\rho_{2}$ ". Put

$$
\mathfrak{S}^{p}\left(\mathcal{A}^{*}\right)=\left\{\rho \in \mathfrak{S}^{m}\left(\mathcal{A}^{*}\right) \mid \rho \text { is a pure state }\right\},
$$

which is called a state space.

An observable $\mathrm{O}:=(X, \mathcal{F}, F)$ in $\mathcal{A}$ (or, a measuring instrument $\mathrm{O}:=(X, \mathcal{F}, F)$ in $\mathcal{A})$ is defined as follows:

i) [field] $X$ is a set, $\mathcal{F}\left(\subseteq 2^{X}\right.$, the power set of $\left.X\right)$ is a field of $X$, that is, “ $\Xi_{1}, \Xi_{2} \in \mathcal{F} \Rightarrow \Xi_{1} \cup \Xi_{2} \in \mathcal{F}$ ” “ $\Xi \in \mathcal{F} \Rightarrow X \backslash \Xi \in \mathcal{F}, \varnothing \in \mathcal{F}$ ”.

ii) [additivity] $F$ is a mapping from $\mathcal{F}$ to $\mathcal{A}$ satisfying: (a): for every $\Xi \in \mathcal{F}$, $F(\Xi)$ is a non-negative element in $\mathcal{A}$ such that $0 \leq F(\Xi) \leq I$, (b): $F(\varnothing)=0$ and $F(X)=I$, where 0 and $I$ is the 0 -element and the identity in $\mathcal{A}$ respectively, (c): for any $\Xi_{1}, \Xi_{2}(\in \mathcal{F})$, it holds that $F\left(\Xi_{1} \cup \Xi_{2}\right)=F\left(\Xi_{1}\right)+F\left(\Xi_{2}\right)-F\left(\Xi_{1} \cap \Xi_{2}\right)$ in $\mathcal{A}$.

\subsection{Axiom 1 [Measurement Axiom] and Axiom 2 [Causality Axiom] in Quantum Language}

Quantum language (1) is composed of two axioms (i.e., Axioms 1 and 2) as follows. With any system $S$, a $C^{\prime}$-algebra $\mathcal{A}(\subseteq B(H))$ can be associated in which the measurement theory (1) of that system can be formulated. A state of the system $S$ is represented by an element $\rho\left(\in \mathfrak{S}^{p}\left(\mathcal{A}^{*}\right)\right)$ and an observable is represented by an observable $O:=(X, \mathcal{F}, F)$ in $\mathcal{A}$. Also, the measurement of the observable $O$ for the system $S$ with the state $\rho$ (or the measurement for the system $S$ with the state $\rho$ by the measuring instrument $O$ ) is denoted by $\mathrm{M}_{\mathcal{A}}\left(\mathrm{O}, S_{[\rho]}\right)$ (or more precisely, $\mathrm{M}_{\mathcal{A}}\left(\mathrm{O}:=(X, \mathcal{F}, F), S_{[\rho]}\right)$ ). An observer can 
obtain a measured value $x(\in X)$ by the measurement $\mathrm{M}_{\mathcal{A}}\left(\mathrm{O}, S_{[\rho]}\right)$.

The Axiom 1 presented below is a kind of mathematical generalization of Born's probabilistic interpretation of quantum mechanics. And thus, it is a statement without reality (i.e., a metaphysical statement).

Now we can present Axiom 1 in the $C$-algebraic formulation as follows.

Axiom 1 [Measurement axiom]. The probability that a measured value $x$ $(\in X)$ obtained by the measurement $\mathrm{M}_{\mathcal{N}}\left(\mathrm{O}:=(X, \mathcal{F}, F), S_{[\rho]}\right)$ (i.e., measurement of the observable $O$ for the system $S$ with the state $\rho$, or measurement for the system $S$ with the state $\rho$ by the measuring instrument $\mathrm{O}$ ) belongs to a set $\Xi(\in \mathcal{F})$ is given by $\rho(F(\Xi))$.

Next, we explain Axiom 2. Let $\mathcal{A}_{1}\left(\subseteq B\left(H_{1}\right)\right)$ and $\mathcal{A}_{2}\left(\subseteq B\left(H_{2}\right)\right)$ be $C$-algebras. A continuous linear operator $\Phi_{1,2}: \mathcal{A}_{2} \rightarrow \mathcal{A}_{1}$ is called a Markov operator, if it satisfies that (i): $\Phi_{1,2}\left(F_{2}\right) \geq 0$ for any non-negative element $F_{2}$ in $\mathcal{A}_{2}$, (ii): $\Phi_{1,2}\left(I_{2}\right)=I_{1}$, where $I_{k}$ is the identity in $\mathcal{A}_{k},(k=1,2)$.

It is clear that the dual operator $\Phi_{1,2}^{*}: \mathcal{A}_{1}^{*} \rightarrow \mathcal{A}_{2}^{*}$ satisfies that $\Phi_{1,2}^{*}\left(\mathfrak{S}^{m}\left(\mathcal{A}_{1}^{*}\right)\right) \subseteq \mathfrak{S}^{m}\left(\mathcal{A}_{2}^{*}\right)$. If it holds that $\Phi_{1,2}^{*}\left(\mathfrak{S}^{p}\left(\mathcal{A}_{1}^{*}\right)\right) \subseteq S^{p}\left(\mathcal{A}_{2}^{*}\right)$, the $\Phi_{1,2}$ is said to be deterministic.

Also note that, for any observable $\mathrm{O}_{2}:=\left(X, \mathcal{F}, F_{2}\right)$ in $\mathcal{A}_{2}$, the $\left(X, \mathcal{F}, \Phi_{1,2} F_{2}\right)$ is an observable in $\mathcal{A}_{1}$.

Now Axiom 2 is presented as follows (For details, see ref. [7].).

Axiom 2 [Causality axiom]. Let $t_{1} \leq t_{2}$. The causality is represented by a Markov operator $\Phi_{t_{1}, t_{2}}: \mathcal{A}_{t_{2}} \rightarrow \mathcal{A}_{t_{1}}$.

Remark 1 In dualistic idealism (i.e., the mainstream of philosophy), we have the two most important problems. One is the mind-body problem (stated in (F) later). Another is the causality problem, i.e., "What is causality?" Note that the solution to the causality problem is given by Axiom 2.

\subsection{The Linguistic Interpretation (=The Manual to Use Axioms 1 and 2)}

In the above, Axioms 1 and 2 are kinds of spells, (i.e., incantation, magic words, metaphysical statements), and thus, it is nonsense to verify them experimentally. Therefore, what we should do is not "to understand" but "to use". After learning Axioms 1 and 2 by rote, we have to improve how to use them through trial and error.

We can do well even if we do not know the linguistic interpretation (=the manual to use Axioms 1 and 2). However, it is better to know the linguistic interpretation, if we would like to make progress quantum language early. I believe that the linguistic interpretation is the true Copenhagen interpretation (cf. ref. [9]).

In what follows I explain the linguistic interpretation:

According to refs. [3] [7], let us explain Figure 1 as follows.

$\left(A_{1}\right)(X)$ : it suffices to understand that "interfere" is, for example, "apply light". (y): perceive the reaction.

That is, "measurement" is characterized as the interaction between "observer" 


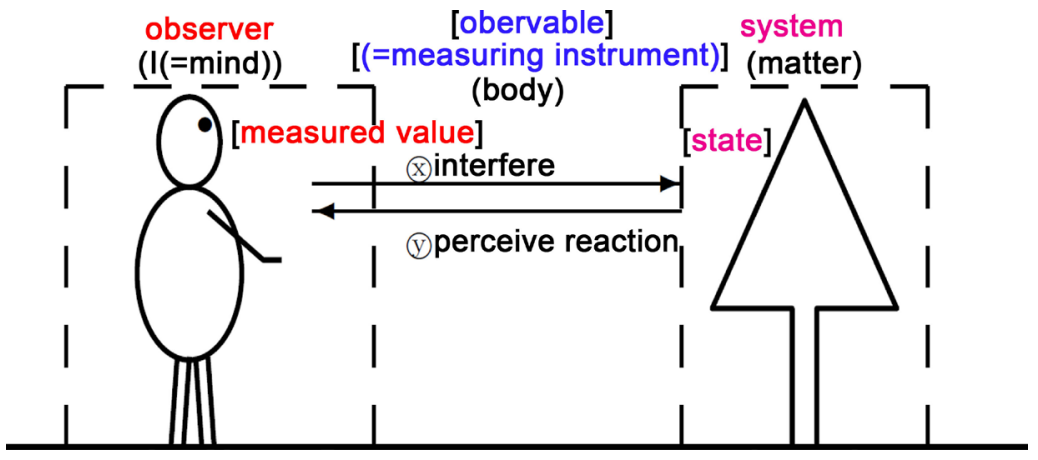

Figure 1. [Descartes Figure]: Image of "measurement $(=\mathrm{x}+\mathrm{y})$ " in mind-matter dualism ( cf. refs. [3] [7]).

and "measuring object (=matter)". However,

$\left(\mathrm{A}_{2}\right)$ in measurement theory (=quantum language), "interaction" must not be emphasized explicitly.

Therefore, in order to avoid confusion, it might better to omit the interaction (x) and (y) in Figure 1.

After all, we think that:

$\left(\mathrm{A}_{3}\right)$ it is clear that there is no measured value without observer (i.e., "I", "mind"). Thus, we consider that measurement theory is composed of three key-words (f. ref. [5]):

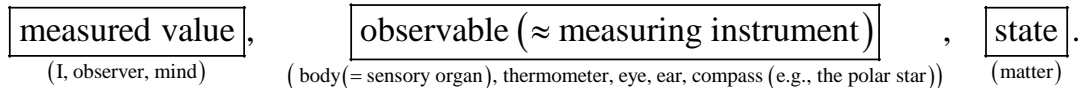

The linguistic interpretation says that:

(B) Only one measurement is permitted. And thus, the state after a measurement is meaningless since it cannot be measured any longer. Thus, the collapse of the wave function is prohibited. We are not concerned with anything after measurement. Strictly speaking, the phrase "after the measurement" should not be used. Also, the causality should be assumed only in the side of system, however, a state never moves. Thus, the Heisenberg picture should be adopted, and thus, the Schrödinger picture should be prohibited.

And so on. That is, I assert that the linguistic interpretation is the true form of so-called Copenhagen interpretation (cf. ref. [9]). For details, see refs. [7] [9].

\subsection{The History of World Description (Philosophy Is Progressive!)}

Let us start from the following figure:

In Figure 2, let us focus on the history of the dualistic idealism in the linguistic world view such as

$$
\text { Plato } \longrightarrow \text { Descartes } \longrightarrow \text { Kocke }
$$

Note that physics (i.e., theories in the realistic world view in Figure 2) surely made progress; on the other hand, there may be various opinions for whether philosophy (3) made progress. In ref. [8], I asserted that, if "(philosophical) progress" is defined by "approaching quantum language", then the following hold: 


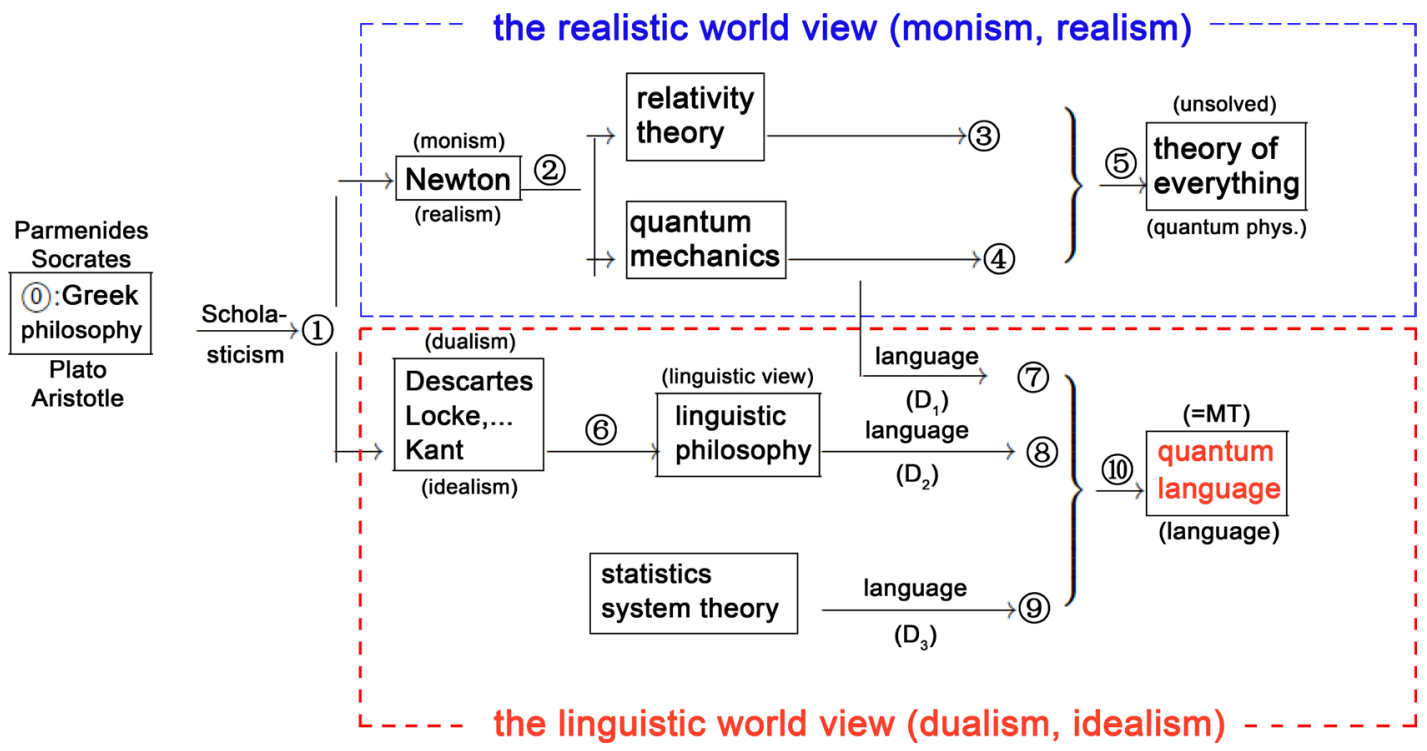

Figure 2. The location of quantum language in the history of the world-description (cf. refs. [3] [7]).

$\left(C_{1}\right)$ the time series (3) does not only represent the change of trend but also represent the course of progress, that is,

Plato $\underset{\text { progress }}{\longrightarrow}$ Descartes $\underset{\text { progress }}{\longrightarrow}$ Locke $\underset{\text { progress }}{\longrightarrow} \underset{\text { Kant }}{\stackrel{\text { progress }}{\longrightarrow} \text { Quantum language }}$

Further, note ( $c f .[7])$ that

$\left(\mathrm{C}_{2}\right)$ Quantum language is the only scientifically successful theory in dualistic idealism.

Hence, I conclude that the following four are equivalent:

$\left(\mathrm{D}_{0}\right)$ to propose quantum language ( $c f$. (10) in Figure 2, refs. [1] [2] [7]).

$\left(D_{1}\right)$ to clarify the Copenhagen interpretation of quantum mechanics ( $\left.c f .7\right)$ in Figure 2, refs. [2] [6] [7]).

$\left(\mathrm{D}_{2}\right)$ to clarify the final goal of the dualistic idealism (cf. (8) in Figure 2, refs. [3] [7] [8]).

$\left(\mathrm{D}_{3}\right)$ to reconstruct statistics in the dualistic idealism (cf. (9) in Figure 2, refs. [4] [7]).

Remark 2. If the progress (4) is true, some may have an opinion that any fundamental problem in dualistic realism should be studied in the latest theory (i.e., quantum language). I think, from the practical point of view, that there is some truth for this opinion, since I assert that quantum language is the only scientifically successful theory in dualistic idealism. In fact, scientists need only quantum language, they do not need others. However, from the viewpoint of enjoying philosophy literally, it is natural that there is objection to this opinion. Although most of the attraction of philosophy may be literary charm in a broad sense ( $c f$. [8]), in this paper I ignore the literary viewpoint of philosophy. That is because the mind-body problem is not a matter of literature.

\section{Three Approaches to the Mind-Body Problem}

If quantum language is the only scientifically successful theory in dualistic ideal- 
ism, it is natural to study the mind-body problem in quantum language. This will be discussed in Section 2.4 (The third approach).

\subsection{The Mind-Body Problem}

Now let us introduce the mind-body problem, which is generally said to be the greatest unsolved problem in dualistic idealism.

In spite that the cogito proposition "I think, therefore I am" is non-sense ( $c f$. refs. [7] [8]), Descartes used it in order to propose Descartes philosophy (i.e., mind-matter dualism). That is, he asserted.

$\left(\mathrm{E}_{1}\right)$ If the existence of "I" is deduced from the cogito proposition, the existence of "matter" (which is perceived by "I") is accepted. And further, the medium of "I" and "matter" is automatically accepted as "body (=sensory organ)".

Therefore, the key-words of Descartes philosophy (=mind-matter dualism) is $\left(\mathrm{E}_{2}\right)$ "I" (="mind"), "body"(="sensory organ"), "matter".

Here, Descartes presented the following problem:

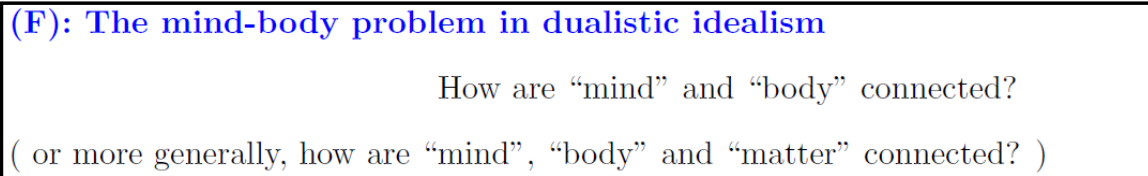

As seen later (i.e., in Section 2.4), this problem is important not only historically but also practically important. That is, any theory in dualistic idealism is useless without the solution to this problem.

\subsection{The First Approach: Brain Scientific Approach}

Although I think, from the philosophical point of view (as mentioned in [9]), that

$\left(\mathrm{G}_{1}\right) \underset{\text { Kant }}{\mathrm{\text {regress }}}$ Husserl ,

some may consider, from the scientific point of view, that

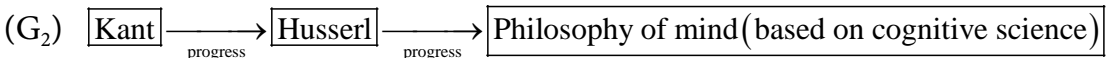

This (i.e., the contradiction of $\left(\mathrm{G}_{1}\right)$ and $\left.\left(\mathrm{G}_{2}\right)\right)$ may be due to the confusion of philosophy and science. Dr. Click (the most noted for being a co-discoverer of the structure of the DNA molecule in 1953 with James Watson) said in his book ("The astonishing hypothesis" [10]) as follows.

$\left(\mathrm{H}_{1}\right)$ You, your joys and your sorrows, your memories and your ambitions, your sense of personal identity and free will, are in fact no more than the behavior of a vast assembly of nerve cells and their associated molecules.

I agree to his opinion $\left(\mathrm{H}_{1}\right)$ (i.e., the denial of the substance dualism), and thus, I believe that the scientific direction $\left(G_{2}\right)$ implies the abandonment of dualistic idealism. Although I fully understand the importance of the scientific aspect of the mind-body problem, this is the work of scientists, not the work of philosophers. I think that the mind-body problem (F) should be within philosophy (particularly, dualistic idealism), and thus, the direction $\left(G_{2}\right)$ is hopeless for the 
mind-body problem (F) in dualistic idealism. Hence, I am not concerned with the first approach (i.e., the study related to the $\left(\mathrm{G}_{2}\right)$ ).

Also, I add that this $\left(\mathrm{H}_{1}\right)$ (i.e., the denial of the substance dualism) and the dualistic idealism (i.e., quantum language) do not contradict each other. That is because quantum language says:

$\left(\mathrm{H}_{2}\right)$ Describe any monistic phenomenon (such as $\left.\left(\mathrm{H}_{1}\right)\right)$ by dualistic language (=quantum language)! (cf. [7] [8]).

\subsection{The Second Approach: Illusory Problem?}

It should be noted that

(I) the term "mind" and "body" in the mind-body problem (F) is ambiguous in Descartes = Kant epistemology.

That is, the sentence "How are 'mind' and 'body' connected?" is meaningless in Descartes = Kant epistemology. Thus, there may be a reason to consider that the mind-body problem (F) is just "what we cannot speak about". Therefore, according to Wittgenstein's famous saying "What we cannot speak about we must pass over in silence" (in [11]), some may conclude that we must speak nothing about the problem (F). That is, the mind-body problem is an illusory problem. However, I think, by $(\mathrm{J})$ and $(\mathrm{K})$ mentioned in the following section, that this second approach is not only non-productive but also wrong.

\subsection{The Third Approach: Quantum Linguistic Solution to the Mind-Body Problem}

It should be noted that

(J) the demarcation problem (i.e., how to distinguish between "what we cannot speak about" and "what we can speak about") depends on language.

For example, the proposition "The earth goes around the sun" cannot be written in mathematics but in the Newtonian mechanical language. Note that both "the limits of my language mean the limits of my world" and "the limits of your language mean the limits of your world" are true. Therefore,

$(\mathrm{K})$ in order to solve the mind-body problem in dualistic idealism, we should create the language in which the mind-body problem can be regarded as "what we can speak about".

Without this challenge $(\mathrm{K})$, we cannot obtain the solution to the mind-body problem (F). In this sense, the second approach in Section 2.3 may be shallow.

This (K) is done as follows. Recall the linguistic turn (cf. refs. [3] [7] [8]):

$$
\text { Descartes }=\text { Kant epistemology } \underset{\text { linguistic turn }}{\longrightarrow} \text { Quantum language }
$$

and recall Figure 1, in which we see the following correspondence:

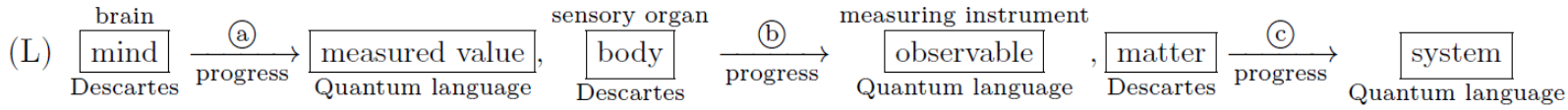

The (a) in (L) may be slightly incomprehensible. However, it suffices to consider "there is no measured value without brain". For example when a needle of a voltmeter just moved, it is only a physical phenomenon. Nevertheless a move- 
ment of this needle is read, and it's sensed by a brain. Then, it for the first time becomes "measured value".

Clearly, "eye" can be regarded as "measuring instrument". Conversely, glasses, microscope, telescope, etc. is a kind of body (=sensory organ). If so, we want to conclude the (b) in (L), that is,

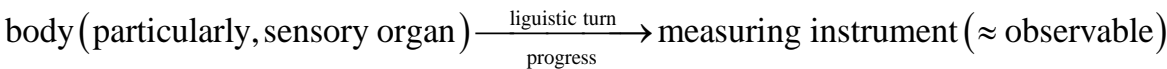

Also, (C) is obvious.

Thus, we can, by (L), consider the linguistic turn of "the epistemological mind-body problem" to "the linguistic mind-body problem" such as

\begin{tabular}{|c|c|c|}
\hline Descartes & & quantum language \\
\hline epistemological mind-body problem & linguistic turn & linguistic mind-body problem \\
\hline
\end{tabular}

Recall Remark 2 (i.e., "any fundamental problem in dualistic realism should be studied in the latest theory (i.e., quantum language)"). Thus I think that it suffices to consider the right hand side of the above (5). Rather than saying, the terms: "mind", "body", "matter", etc. can be for the first time understood after the translation (5) to quantum language. Hence, the mind-body problem in dualistic idealism is easily solved by Axiom 1 as follows:

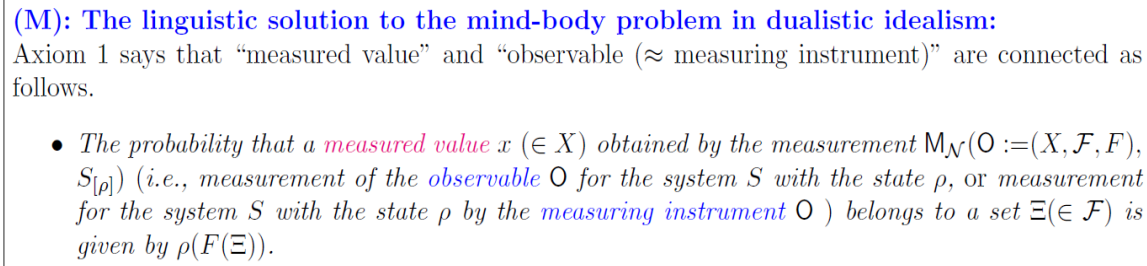

- The probability that a measured value $x(\in X)$ obtained by the measurement $\mathrm{M}_{\mathcal{N}}(\mathrm{O}:=(X, \mathcal{F}, F)$, $\left.S_{[\rho]}\right)$ (i.e., measurement of the observable $\mathrm{O}$ for the system $S$ with the state $\rho$, or measurement for the system $S$ with the state $\rho$ by the measuring instrument $O$ ) belongs to a set $\Xi(\in \mathcal{F})$ is given by $\rho(F(\Xi))$.

That is, we conclude that

$(\mathrm{N})$ to solve the mind-body problem $\Leftrightarrow$ to propose Axiom 1 (measurement).

\section{Conclusions}

In this paper, I am not concerned with the literary aspect of philosophy since the mind-body problem is not a matter of literature. If Descartes = Kant epistemology (which is not scientifically successful) made progress to quantum language (which is scientifically successful), the mind-body problem should be reconsidered in quantum language. This was done in Section 2.4, and the solution (M) to the mind-body problem was obtained. That is, I conclude, by Remark 1 and the above $(\mathrm{N})$, that

(O) to propose quantum language:

$\Leftrightarrow$ to find Axiom 1 (measurement) and Axiom 2 (causality);

$\Leftrightarrow$ to solve the mind-body problem and the causality problem.

I believe that Kant's dream was to build a firm theory in dualistic idealism. The first approach in Section 2.2 is not philosophical (i.e., a scientific direction mentioned in $\left.\left(\mathrm{H}_{1}\right)\right)$, and the second approach is shallow. Therefore, I am convinced that my proposal (i.e., $(\mathrm{M})$ or equivalently $(\mathrm{O})$ ) is the final solution to the 
mind-body problem in dualistic idealism. If the above $(\mathrm{O})$ is true, I can understand the reason why the mind-body problem is generally said to be the most important problem in dualistic idealism.

I hope that my proposal will be examined from various points of view ${ }^{1}$.

\section{References}

[1] Ishikawa, S. (2006) Mathematical Foundations of Measurement Theory. Keio University Press Inc. Tokyo, 335 p. http://www.keio-up.co.jp/kup/mfomt/

[2] Ishikawa, S. (2011) A New Interpretation of Quantum Mechanics. Journal of Quantum Information Science, 1, 35-42. https://doi.org/10.4236/jqis.2011.12005 http://www.scirp.org/journal/PaperInformation.aspx?paperID=7610

[3] Ishikawa, S. (2012) Quantum Mechanics and the Philosophy of Language: Reconsideration of Traditional Philosophies. Journal of Quantum Information Science, 2, 2-9. http://www.scirp.org/journal/PaperInformation.aspx?paperID=18194 https://doi.org/10.4236/jqis.2012.21002

[4] Ishikawa, S. (2012) A Measurement Theoretical Foundation of Statistics. Applied Mathematics, 3, 283-292. https://doi.org/10.4236/am.2012.33044 http://www.scirp.org/Journal/PaperInformation.aspx?PaperID=18109

[5] Ishikawa, S. (2012) Measurement Theory in the Philosophy of Science. http://arxiv.org/abs/1209.3483

[6] Ishikawa, S. (2015) Linguistic Interpretation of Quantum Mechanics; Projection Postulate. Journal of Quantum Information Science, 5, 150-155. https://doi.org/10.4236/jqis.2015.54017 http://www.scirp.org/Journal/PaperInformation.aspx?PaperID=62464

[7] Ishikawa, S. (2016) Linguistic Interpretation of Quantum Mechanics: Quantum Language Version 2. Research Report, Shiho-Shuppan Publisher, Tsukuba, KSTSRR-16/001, 416 p. http://www.math.keio.ac.jp/academic/research_pdf/report/2016/16001.pdf

[8] Ishikawa, S. (2017) History of Western Philosophy from the Quantum Theoretical Point of View Version 2. Research Report, Department of Mathematics, Keio University, Yokohama, KSTS-RR-17/004, 139 p.

http://www.math.keio.ac.jp/academic/research_pdf/report/2017/17004.pdf

[9] Howard, D. (2004) Who Invented the "Copenhagen Interpretation"? A Study in Mythology. Philosophy of Science, 71, 669-682. https://doi.org/10.1086/425941

[10] Click, F. (1994) The Astonishing Hypothesis: The Scientific Search for the Soul. Charles Scribner's Sons., New York.

[11] Wittgenstein, L. (1921) Tractatus Logico-Philosophicus. Routledge and Kegan Paul, Oxford, London.

${ }^{1}$ For the further information of quantum language, see http://www.math.keio.ac.jp/ ishikawa/indexe.html. 
Submit or recommend next manuscript to SCIRP and we will provide best service for you:

Accepting pre-submission inquiries through Email, Facebook, LinkedIn, Twitter, etc. A wide selection of journals (inclusive of 9 subjects, more than 200 journals)

Providing 24-hour high-quality service

User-friendly online submission system

Fair and swift peer-review system

Efficient typesetting and proofreading procedure

Display of the result of downloads and visits, as well as the number of cited articles Maximum dissemination of your research work

Submit your manuscript at: http://papersubmission.scirp.org/

Or contact jqis@scirp.org 